\title{
Interactive Complex Granules
}

\author{
Andrzej Jankowski ${ }^{1}$, Andrzej Skowron ${ }^{2}$, and Roman Swiniarski ${ }^{3 \star}$ \\ 1 Institute of Computer Science, Warsaw University of Technology \\ Nowowiejska 15/19, 00-665 Warsaw, Poland \\ a.jankowski@ii.pw.edu.pl \\ 2 Institute of Mathematics, The University of Warsaw \\ Banacha 2, 02-097 Warsaw, Poland \\ skowron@mimuw.edu.pl \\ 3 Department of Computer Science, San Diego State University \\ 5500 Campanile Drive San Diego, CA 92182, USA \\ and \\ Institute of Computer Science Polish Academy of Sciences \\ Jana Kazimierza 5, 01-248 Warsaw, Poland \\ rswiniarski@mail.sdsu.edu \\ As far as the laws of mathematics refer to reality, \\ they are not certain; and as far as they are certain, \\ they do not refer to reality. \\ - Albert Einstein ([2]) \\ Constructing the physical part of the theory and unifying it \\ with the mathematical part should be considered as one of \\ the main goals of statistical learning theory \\ - Vladimir Vapnik \\ ([24], Epilogue: Inference from sparse data, p. 721)
}

\begin{abstract}
Information granules (infogranules, for short) are widely discussed in the literature. In particular, let us mention here the rough granular computing approach based on the rough set approach and its combination with other approaches to soft computing. However, the issues related to interactions of infogranules with the physical world and to perception of interactions in the physical world by infogranules are
\end{abstract}

* This work was supported by the Polish National Science Centre grants 2011/01/B/ ST6/03867, 2011/01/D/ST6/06981, and 2012/05/B/ST6/03215 as well as by the Polish National Centre for Research and Development (NCBiR) under the grant SYNAT No. SP/I/1/77065/10 in frame of the strategic scientific research and experimental development program: "Interdisciplinary System for Interactive Scientific and Scientific-Technical Information" and the grant No. O ROB/0010/ 03/001 in frame of the Defence and Security Programmes and Projects: "Modern engineering tools for decision support for commanders of the State Fire Service of Poland during Fire \& Rescue operations in the buildings" 
not well elaborated yet. On the other hand the understanding of interactions is the critical issue of complex systems. We propose to model complex systems by interactive computational systems (ICS) created by societies of agents. Computations in ICS are based on complex granules (c-granules, for short). In the paper we concentrate on some basic issues related to interactive computations based on c-granules performed by agents in the physical world.

Key words: granular computing, rough set, interaction, information granule, physical object, complex granule, interactive computational system

\section{Introduction}

Granular Computing (GC) is now an active area of research (see, e.g., [16]). Objects we are dealing with in GC are information granules (or infogranules, for short). Such granules are obtained as the result of information granulation [26, 28]:

Information granulation can be viewed as a human way of achieving data compression and it plays a key role in implementation of the strategy of divide-and-conquer in human problem-solving.

The concept of granulation is rooted in the concept of a linguistic variable introduced by Lotfi Zadeh in 1973 [25]. Information granules are constructed starting from some elementary ones. More compound granules are composed of finer granules that are drawn together by indistinguishability, similarity, or functionality [27].

Computations on granules should be interactive. This requirement is fundamental for modeling of complex systems [3]. For example, in [13] this is expressed as follows

[...] interaction is a critical issue in the understanding of complex systems of any sorts: as such, it has emerged in several well-established scientific areas other than computer science, like biology, physics, social and organizational sciences.

Interactive Rough Granular Computing (IRGC) is an approach for modeling interactive computations (see, e.g., $[17,19-23])$. Computations in IRGC are progressing by interactions represented by interactive information granules. In particular, interactive information systems (IIS) are dynamic granules used for representing the results of the agent interaction with the environments. IIS can be also applied in modeling of more advanced forms of interactions such as hierarchical interactions in layered granular networks or generally in hierarchical modeling. The proposed approach $[17,19-23]$ is based on rough sets but it can be combined with other soft computing paradigms such as fuzzy sets or evolutionary computing, and also with machine learning and data mining techniques. 
The notion of the highly interactive granular system is clarified as the system in which intrastep interactions [4] with the external as well as with the internal environments take place. Two kinds of interactive attributes are distinguished: perception attributes, including sensory ones and action attributes.

In this paper we extend the existing approach by introducing complex granules (c-granules) making it possible to model interactive computations performed by agents. Any c-granule consists of three components, namely soft_suit, link_suit and hard_suit. These components are making it possible to deal with such abstract objects from soft_suit as infogranules as well as with physical objects from hard_suit. The link_suit of a given c-granule is used as a kind of c-granule interface for expressing interaction between soft_suit and and hard_suit. Any agent operates in a local world of c-granules. The agent control is aiming to control computations performed by c-granules from this local world for achieving the target goals. Actions (sensors or plans) from link_suits of c-granules are used by the agent control in exploration and/or exploitation of the environment on the way to achieve their targets. C-granules are also used for representation of perception by agents of interactions in the physical world. Due to the bounds of the agent perception abilities usually only a partial information about the interactions from physical world may be available for agents. Hence, in particular the results of performed actions by agents can not be predicted with certainty.

In Section 2 a general structure of c-granules is described and some illustrative examples are included. Moreover, some preliminary concepts related to agents performing computations on c-granules are discussed. In Section 3 the agent architecture is outlined. Societies of agents and communication languages are discussed shortly in Section 4.

This paper is a step in the realization of the Wisdom Technology (WisTech) programme $[6-8]$.

\section{Complex Granules and Physical World}

We define the basic concepts related to c-granule relative to a given agent $a g$. We assume that the agent $a g$ has access to a local clock making it possible to use the local time scale. In this paper we consider discrete linear time scale.

We distinguish several kinds of objects in the environment in which the agent ag operates:

- physical objects (called also as hunks of matter, or hunks, for short) [5] such as physical parts of agents or robots, specific media for transmitting information; we distinguish hunks called as artifacts used for labeling other hunks or stigmergic markers used for indirect coordination between agents or actions [9]; note that hunks may change in time and are perceived by the agent $a g$ as dynamic (systems) processes; any hunk $h$ at the local time $t$ of $a g$ is represented by the state $s t_{h}(t)$; the results of perception of hunk states by agent $a g$ are represented by value vector of relevant attributes (features);

- complex granules (c-granules, for short) consisting of three parts: soft_suit, link_suit, and hard_suit (see Figure 1); c-granule at the local time $t$ of $a g$ is 
denoted by $G$; $G$ receives some inputs and produces some outputs; inputs and outputs of c-granule $G$ are c-granules of the specified admissible types; input admissible type is defined by some input preconditions and the output admissible type is defined by some output postconditions, there are distinguished inputs (outputs) admissible types which receive (send) c-granules from (to) the agent $a g$ control;

- $G$ soft_suit consists of

1. $G$ name, describing the behavioral pattern description of the agent $a g$ corresponding to the name used by agent for identification of the granule,

2. $G$ type consisting of the types of inputs and outputs of $G$ c-granule,

3. $G$ status (e.g., active, passive),

4. $G$ information granules (infogranules, for short) in mental imagination of the agent consisting, in particular of $G$ specification, $G$ implementation and manipulation method(s); any implementation distinguished in infogranule is a description in the agent $a g$ language of transformation of input c-granules of relevant types into output c-granules of relevant types, i.e., any implementation defines an interactive computation which takes as input c-granules (of some types) and produces some c-granules (of some types); inputs for c-granules can be delivered by the agent ag control (or by other c-granules), we also assume that the outputs produced by a given c-granule depend also on interactions of hunks pointed out by link_suite as well as some other hunks from the environment - in this way the semantics of c-granules is established;

- $G$ link_suit consists of

1. a representation of configuration of hunks at time $t$ (e.g., mereologies of parts in the physical configurations perceived by the agent $a g$ );

2. links from different parts of the configuration to hunks;

3. $G$ links and $G$ representations of elementary actions; using these links the agent ag may perform sensory measurement or/and actions on hunks; in particular, links are pointing to the sensors or effectors in the physical world used by the considered c-granule; using links the agent $a g$ may, e.g., fix some parameters of sensors or/and actions, initiate sensory measurements or/and action performance; we also assume that using these links the agent ag may encode some information about the current states of the observed hunks by relevant information granules;

- $G$ hard_suit is created by the environment of interacting hunks encoding $G$ soft_suit, $G$ link_suit and implementing $G$ computations;

- soft_suit and link_suit of $G$ are linked by $G$ links for interactions between the $G$ hunk configuration representation and $G$ infogranules;

- link_suit and hard_suit are linked by $G$ links for interactions between the $G$ hunk configuration representation and hunks in the environment.

The interactive processes during transforming inputs of c-granules into outputs of c-granules is influenced by 
1. interaction of hunks pointed out by link_suit;

2. interaction of pointed hunks with relevant parts of configuration in link_suit.

Agent can establish, remember, recognize, process and modify relations between c-granules or/and hunks.

A general structure of c-granules is illustrated in Figure 1.

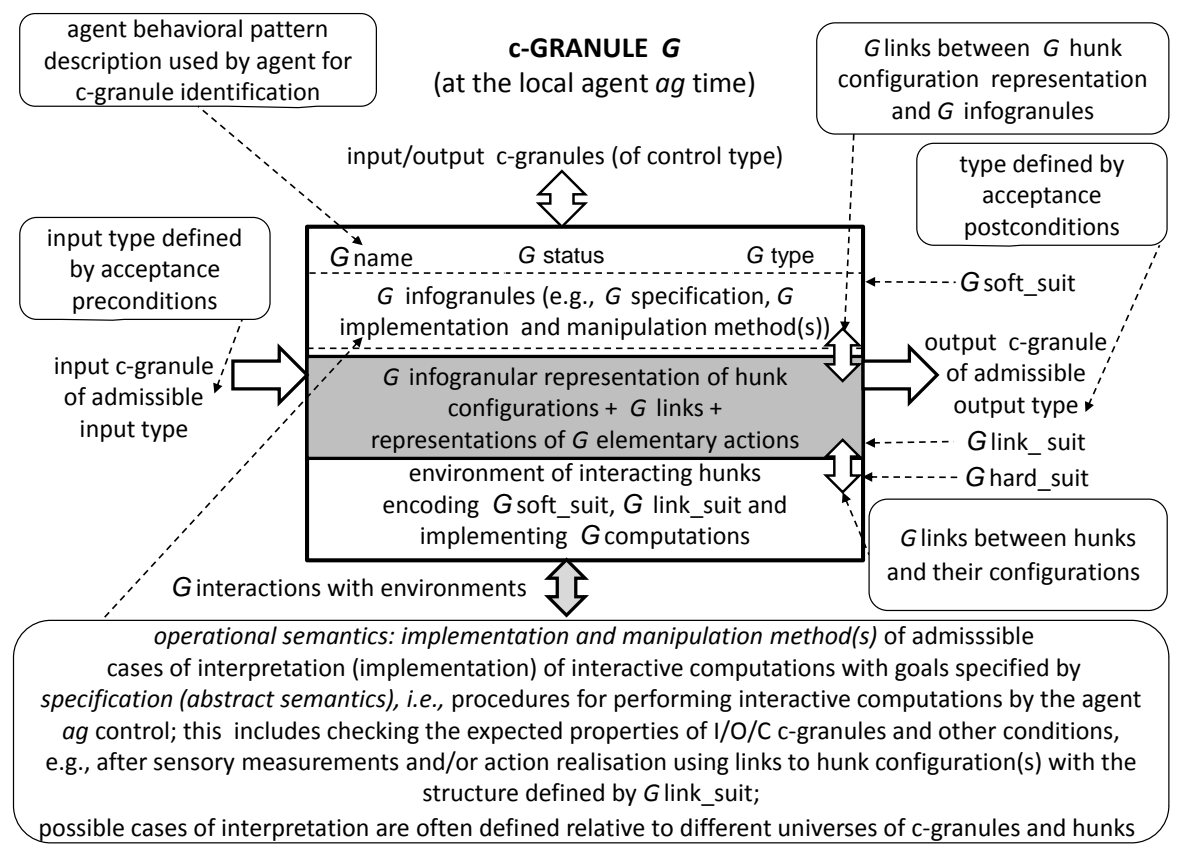

Fig. 1. General structure of c-granules

In Figure 2 we illustrate how the abstract definition of operation from soft_link interacts with other suits of c-granule. It is necessary to distinguish two cases. In the first case, the results of operation $\otimes$ realized by interaction of hunks are consistent with the specification in the suit_link. In the second case, the result specified in the soft_suit can be treated only as an estimation of the real one which may be different due to the unpredictable interactions in the hard_suit. Figure 3 illustrates c-granules corresponding to sensory measurement. Note that in this case, the parameters fixed by the agent control may concern sensor selection, selection of the object under measurement by sensor and selection of sensor parameters. They are interpreted as actions selected from the link_suit. In the perception of configuration of hunks of c-granule are distinguished infogranules representing sensor, object under measurement and the configuration itself. The links selected by the agent control represent relations between states of hunks and infogranules corresponding to them in the link_suit. 


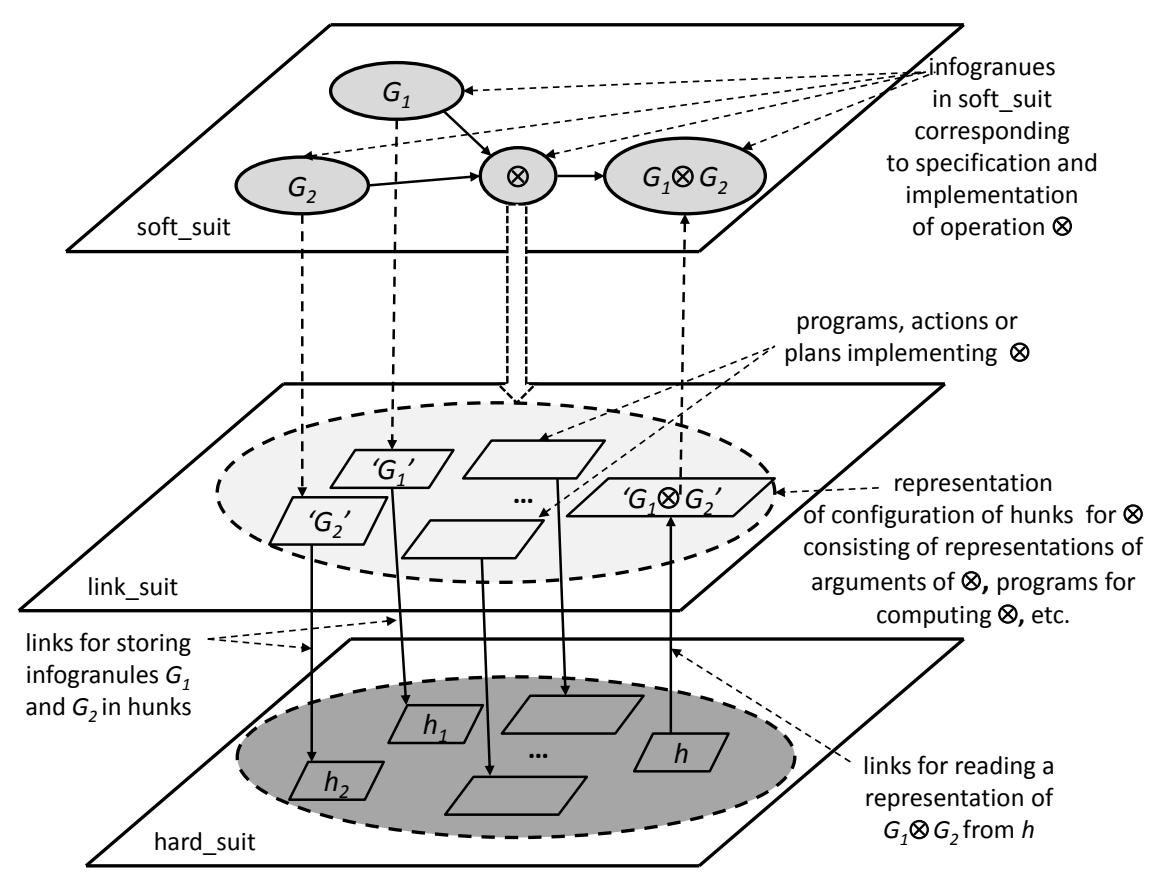

Fig. 2. Explanation of roles of different suits of a c-granule for operation $\otimes$

Figure 4 illustrates how an interactive information (decision) system is created and updated during running of c-granule implementation according to scenario(s) defined in the soft_suit and related G links. Such information (decision) systems are used for recording information about the computation steps during c-granule implementation run. Note that the structure of this information system is different from the classical definition $[14,15,18]$. In particular, this system is open because of links to physical objects as well as interactions are changing (often in unpredictable way) in time. In our approach, the agent can be also interpreted as c-granule. However, this is a c-granule of higher order with embedded control. One can also consider another situation when the c-granules are autonomous but this is out of scope of this article. Instead of this one can consider interactions in societies of agents. We assume that for any agent $a g$ there is distinguished a family of elementary c-granules and constructions on c-granules leading to more compound c-granules. The agent $a g$ is using the constructed granules for modeling attention and interaction with the environment. Note that for any new construction on elementary granules (such as network of c-granules) should be defined the corresponding c-granule. This c-granule should have appropriate soft_suit, link_suit and hard_suit so that the constructed c-granule will satisfy the conditions of the new c-granule construction specification. Note that one of the constraints on such construction may follow from the interactions which the agent $a g$ will have at the disposal in the uncertain environment. 


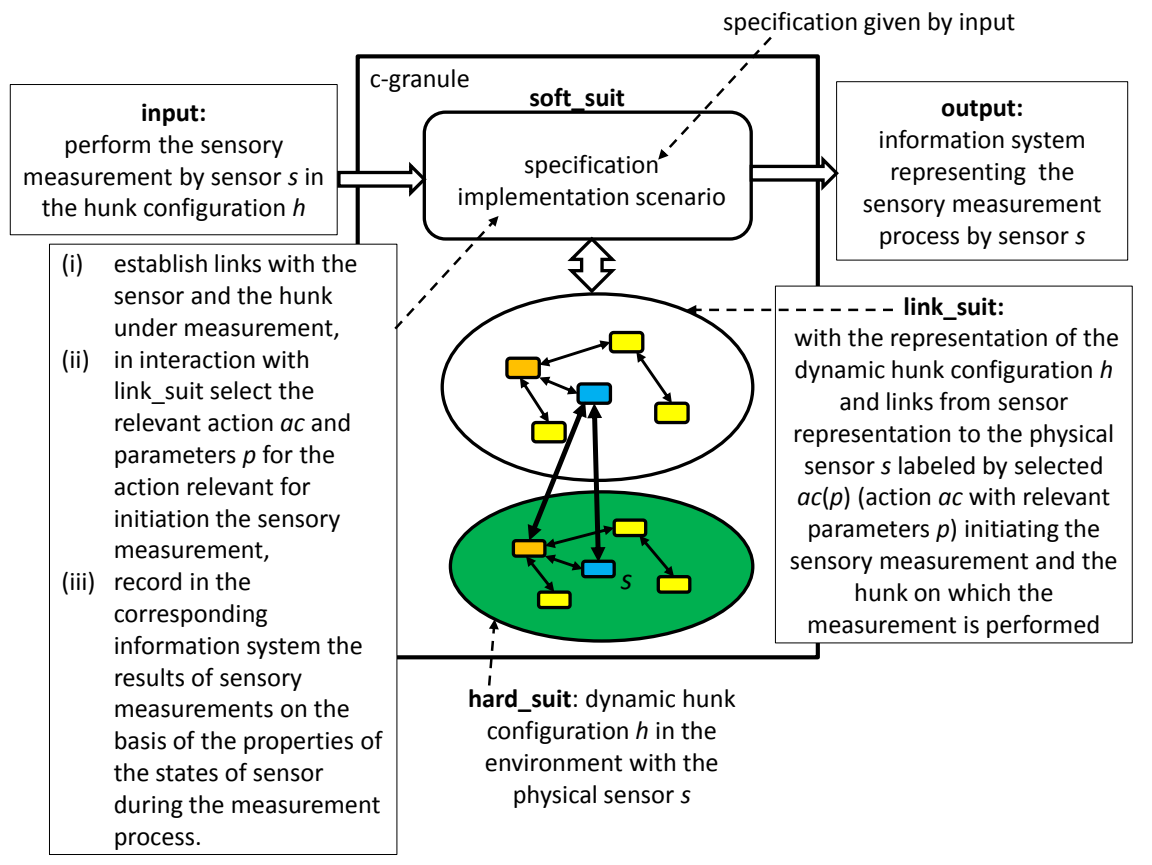

Fig. 3. Interactions caused by sensors

\section{Agent Architecture Framework}

Agents may be treated as generalized c-granules with embedded control structure.

Any agent $a g$ is defined over several classes of c-granules. Among them are:

- senbot (sensory bot) - class of c-granules representing possible states of the agent sensory measurements with at most one distinguished c-granule at the local time moment $t$ of agent $a g$;

- imbot (imagination bot)- class of all possible c-granules which can be constructed by the agent $a g$ from sensory measurements with at most one distinguished c-granule at the local time moment $t$ of agent $a g$;

- embot (emotional bot)- subclass of imbot class representing emotional concepts of the agent $a g$;

- nebot (needs bot)- subclass of imbot class representing concepts of the agent ag needs;

- enabot (environment action bot) - subclass of imbot class specifying the agent $a g$ elementary actions in the environment;

- imobot (imagination operation bot) - subclass of imbot class specifying the agent $a g$ elementary operations (different from elementary actions) on cgranules from imbot; 


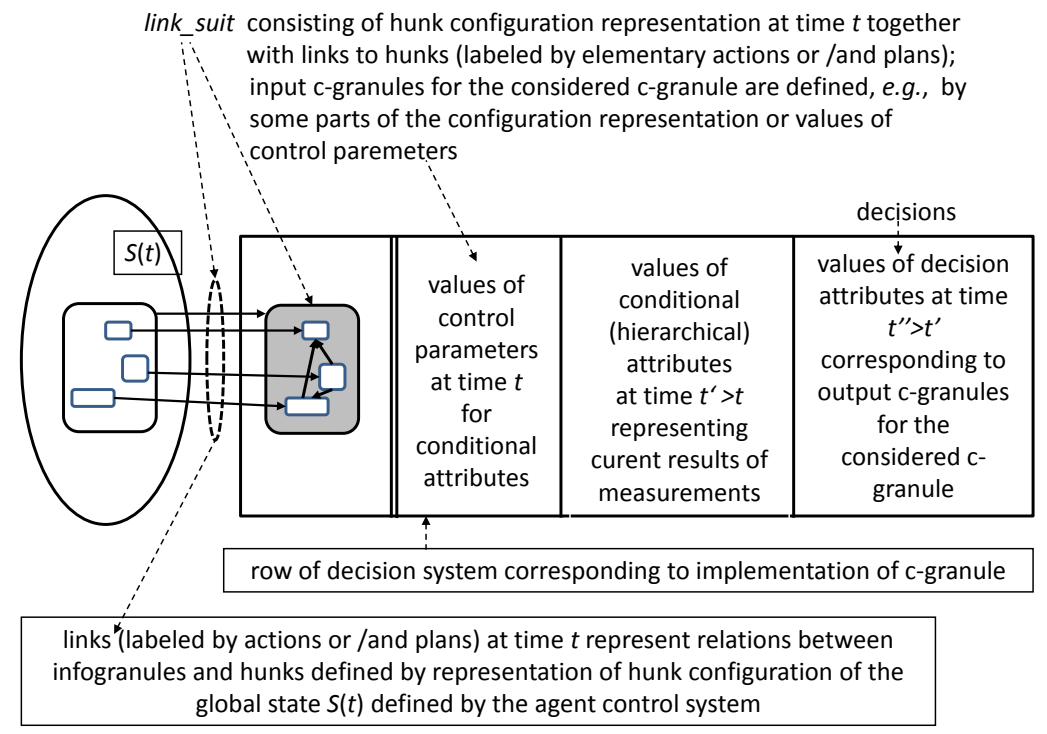

Fig. 4. Example: Row of interactive information (decision) system corresponding to registration of computation of c-granule according to implementation scenario

- abot (attention bot) - subclass of imbot class representing c-granules currently under attention by the agent $a g$;

- activebot - subclass of imbot class representing c-granules currently active;

- passivebot - subclass of imbot class representing c-granules currently passive;

- metbot (method bot) - subclass of imbot representing methods of manipulation on c-granules (construction, destruction, modification, join, classifiers construction);

- metabot (method adaptation bot) subclass of imbot representing c-granules used for adaptation or/and modification of the given methods of manipulation on c-granules.

The language of c-granule names consists of

- set of names of existing c-granules;

- set of names of new generated c-granules.

Types of objects relative to c-granules in imbot:

- set of types of existing c-granules;

- set of types of new generated c-granules.

There are some distinguished c-granules of the agent ag:

- Meaning relation (Mean) - a distinguished c-granule representing a relation between c-granules and their names. 
- Type relation (TypeMean) - a distinguished c-granule representing a relation between c-granules and their types.

- Reference relation (Ref) - a distinguished c-granule representing a relation between c-granules and 'related' names.

- Jbot (Judgment bot) - a distinguished c-granule representing actual collection of strategies of approximate reasoning used by the agent $a g$ for judgment and risk assessment in the current environment and agent situation.

- Cobot (control bot) - a distinguished c-granule representing actual collection of strategies of approximate reasoning used by the agent $a g$ for control, adaptation, and modification of all the agent ag c-granules.

- Metacobot (meta-control bot) - a distinguished c-granule representing actual collection of strategies of approximate reasoning used by the agent $a g$ for cobot control, adaptation, and modification.

The generalized c-granules corresponding to agents are defined using also the above classes of c-granules for defining corresponding suits of such generalized c-granules. The details of such construction will be presented in our next papers. Here, we would like to note only that there is a quite general approach for defining new c-granules from the simpler already defined.

Figure 5 illustrates an idea of transition relation related to a given agent $a g$. The relation is defined between configurations of $a g$ at time $t$ and the measurement time next to $t$. A configuration of $a g$ at time $t$ consists of all configurations of c-granules existing at time $t$. A configuration of c-granule $G$ at time $t$ consists of $G$ itself as well as all c-granules selected on the basis of links in the link_suit of $G$ at time $t$. These are, in particular all c-granules pointed by links corresponding to the c-granules stored in the computer memory during the computation process realised by c-granule as well as c-granules corresponding to perception at time $t$ of the configuration of hunks at time $t$.

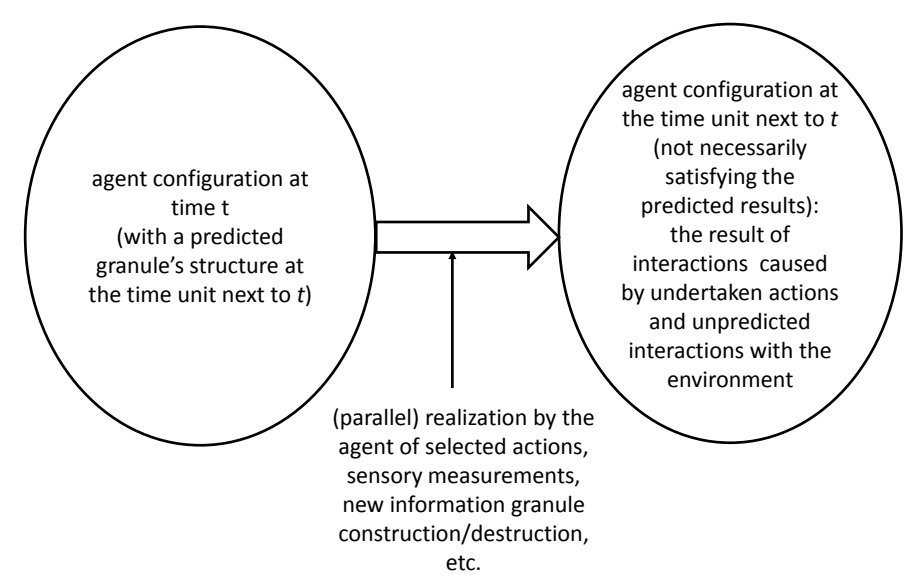

Fig. 5. Transiton relation of the agent $a g$ 


\section{Societies of Agents and Communication Languages}

We assume that the agents can perceive behavioral patterns of other agents of their groups and based on this they can try to exchange some messages [10]. It is worthwhile to mention that at the beginning agents do not have common understanding of the meaning of such messages. In the consequence, this leads to misunderstanding, not comfortable situation for agents (in terms of hierarchy of their needs represented by nebot). However, after series of trials they have a chance to set up common meaning of some behavioral patterns. In other words, they start to create common c-granules which use agreed links to other hunks or infogranules and also descriptions of some details about actions related to meaning or methods of implementation of the infogranule contents. For example, at the beginning the messages could be linked to warning situations or to identifications of some sources for satisfiability of some agent needs. This kind of simple messages could be passed by very simple behavioral pattern. Next, based on these very simple behavioral patterns the agents can develop more compound messages related to c-granules corresponding to common plans of cooperation of group of agents or/and competition with other groups of agents. This very general framework could be implemented in many ways using different AI paradigms. Especially, many models from Natural Computing could be quite helpful (e.g., modification of cellular automata or evolutionary programming). However, our proposal is to implement this general scheme by agents having soft_suit and link_suit built up on the hierarchies of interactive information (decision) systems linked to configurations of hunks. Starting from the simplest case when we have just one attribute and one message to be passed up to quite complex system this approach based on rough sets is quite convenient for implementation by computers well prepared for manipulation on tables of data.

It has to be underlined that the behavioral patterns are complex vague concepts. Hence, some advanced methods for approximation of these concepts should be used. Usually these methods are based on hierarchical learning (see, e.g., [11, 1]). Note that often in satisfiability checking for vague concept, actions or/and plans are used. In the rough set approach it is important to remember that the attribute values are given only for some examples from reality. Moreover, if we use a large number of attributes or/ and hierarchical learning this will not guarantee the exact description of reality in terms of perceived vague concepts.

Languages of agents consist of partial descriptions of situations (or their indiscernibility or similarity classes) perceived by agents as well as description of approximate reasoning schemes about the situations and their changes by actions and /or plans. The situations may be represented in hierarchical modeling by structured objects (e.g., relational structures over attribute value vectors or/and indiscernibility (similarity classes) of such structures). In reasoning about the situation changes one should take into account that the predicted actions or/and

planes may depend not only on the changes of past situations but also on the performed actions and plane in the past. This is strongly related to the idea of perception pointed out in [12]: 
The main idea of this book is that perceiving is a way of acting. It is something we do. Think of a blind person tap-tapping his or her way around a cluttered space, perceiving that space by touch, not all at once, but through time, by skillful probing and movement. This is or ought to be, our paradigm of what perceiving is.

Figure 6 illustrates this idea.

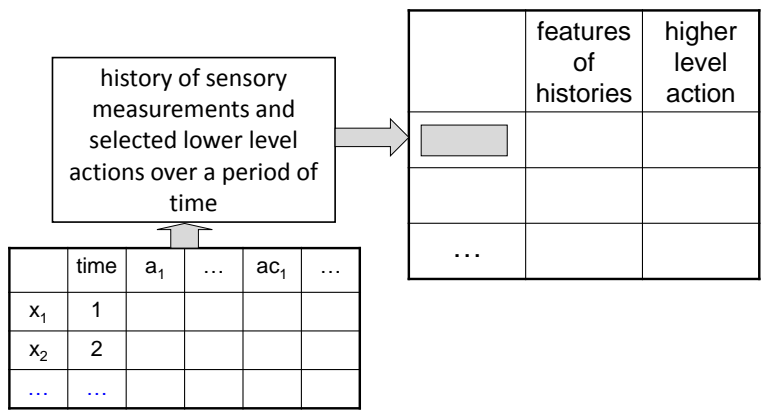

Fig. 6. Action in perception.

Note that the expression of the language may be used without its 'support' in corresponding link_suit and hard_suit of c-granules under the assumption that there are fixed coding methods between expressions and hunks by the agent. However, the languages should contain more general expressions for communication usually requiring the usage of expressions representing classes of hunks rather than single hunks. This follows from the fact that the agents have bounded abilities for discernibility of perceived objects. In our approach the situations and reasoning schemes about situations are represented by c-granules.

Note that different behavioral patterns may be indiscernible relative to the set of attributes used by the agent. Hence, it follows that the agents perceive objects belonging to the same indiscernibility or/and similarity class in the same way. This is an important feature making it possible to use generalization by agents. For example, the situations classified by a given set of characteristic functions of induced classifiers (used as attributes) may be indiscernible. On the other hand, a new situation unseen so far may be classified to indiscernibility classes which allows agents to make generalizations. The new names created by agents are names of new structured objets or their indiscernibility (similarity) classes.

Agents should be equipped with adaptation strategies for discovery of new structured objects and their features (attributes). This is the consequence of the fact that the agents are dealing with vague concepts. Hence, the approximations of these concepts represented by the induced classifiers evolve with changes in uncertain data and imperfect knowledge. 


\section{Conclusions and Future Research}

The outlined research on the nature of interactive computations is crucial for understanding complex systems. Our approach is based on complex granules (c-granules) performing computations through interaction with physical objects (hunks). Computations of c-granules are controlled by the agent control. More compound c-granules create agents and societies of agents. Other issues outlined in this paper such as interactive computations performed by societies for agents, especially communication language evolution and risk management in interactive computations will be discussed in more detail in our next papers.

\section{References}

1. J. Bazan. Hierarchical classifiers for complex spatio-temporal concepts. Transactions on Rough Sets IX: Journal Subline LNCS 5390 (2008) 474-750.

2. A. Einstein. Geometrie und Erfahrung (Geometry and Experience). Julius Springer, Berlin, 1921.

3. D. Goldin, S. Smolka, P. Wegner (Eds.). Interactive Computation: The New Paradigm. Springer, 2006.

4. Y. Gurevich. Interactive algorithms 2005. In: Goldin et al. [3], pp. 165-181.

5. M. Heller. The Ontology of Physical Objects. Four Dimensional Hunks of Matter. Cambridge Studies in Philosophy, Cambridge University Press, Cambridge, UK, 1990.

6. A. Jankowski, A. Skowron. A wistech paradigm for intelligent systems. Transactions on Rough Sets VI: Journal Subline LNCS 4374 (2007) 94-132.

7. A. Jankowski, A. Skowron. Wisdom technology: A rough-granular approach. In: M. Marciniak, A. Mykowiecka (Eds.), Bolc Festschrift, Springer, Heidelberg, Lectures Notes in Computer Science, vol. 5070. 2009, pp. 3-41.

8. A. Jankowski, A. Skowron. Practical Issues of Complex Systems Engineering: Wisdom Technology Approach. Springer, Heidelberg, 2014. (in preparation).

9. L. Marsh. Stigmergic epistemology, stigmergic cognition. Journal Cognitive Systems 9 (2008) 136-149.

10. M. Minsky. Emotion Machine: Commonsense Thinking, Artificial Intelligence, and the Future of the Mind. Simon \& Schuster, New York, 2006.

11. S. H. Nguyen, J. Bazan, A. Skowron, H. S. Nguyen. Layered learning for concept synthesis. Transactions on Rough Sets I: Journal Subline LNCS 3100 (2004) 187208.

12. A. Noë. Action in Perception. MIT Press, 2004.

13. A. Omicini, A. Ricci, M. Viroli. The multidisciplinary patterns of interaction from sciences to computer science. In: Goldin et al. [3], pp. 395-414.

14. Z. Pawlak. Information systems - theoretical foundations. Information Systems 6 (1981) 205-218.

15. Z. Pawlak. Rough Sets: Theoretical Aspects of Reasoning about Data, System Theory, Knowledge Engineering and Problem Solving, vol. 9. Kluwer Academic Publishers, Dordrecht, The Netherlands, 1991.

16. W. Pedrycz, S. Skowron, V. Kreinovich (Eds.). Handbook of Granular Computing. John Wiley \& Sons, Hoboken, NJ, 2008.

17. A. Skowron, J. Stepaniuk, R. Swiniarski. Modeling rough granular computing based on approximation spaces. Information Sciences 184 (2012) 20-43. 
18. A. Skowron, Z. Suraj (Eds.). Rough Sets and Intelligent Systems. Professor Zdzislaw Pawlak in Memoriam. Series Intelligent Systems Reference Library, Springer, 2013.

19. A. Skowron, M. Szczuka. Toward interactive computations: A rough-granular approach. In: J. Koronacki, Z. Raś, S. Wierzchoń, J. Kacprzyk (Eds.), Advances in Machine Learning II: Dedicated to the Memory of Professor Ryszard S. Michalski, Springer, Heidelberg, Studies in Computational Intelligence, vol. 263. 2009, pp. 23-42.

20. A. Skowron, P. Wasilewski. Information systems in modeling interactive computations on granules. In: M. Szczuka, M. Kryszkiewicz, S. Ramanna, R. Jensen, Q. Hu (Eds.), Proceedings of RSCTC 2010, Springer, Heidelberg, Lectures Notes in Computer Science, vol. 6086. 2010, pp. 730-739.

21. A. Skowron, P. Wasilewski. Information systems in modeling interactive computations on granules. Theoretical Computer Science 412(42) (2011) 5939-5959.

22. A. Skowron, P. Wasilewski. Toward interactive rough-granular computing. Control \& Cybernetics 40(2) (2011) 1-23.

23. A. Skowron, P. Wasilewski. Interactive information systems: Toward perception based computing. Theoretical Computer Science 454 (2012) 240-260.

24. V. Vapnik. Statistical Learning Theory. John Wiley \& Sons, New York, NY, 1998.

25. L. A. Zadeh. Outline of a new approach to the analysis of complex systems and decision processes. IEEE Trans. on Systems, Man and Cybernetics SMC-3 (1973) $28-44$.

26. L. A. Zadeh. Fuzzy sets and information granularity. In: Advances in Fuzzy Set Theory and Applications, North-Holland, Amsterdam. 1979, pp. 3-18.

27. L. A. Zadeh. Toward a theory of fuzzy information granulation and its centrality in human reasoning and fuzzy logic. Fuzzy Sets and Systems 90 (1997) 111-127.

28. L. A. Zadeh. A new direction in AI: Toward a computational theory of perceptions. AI Magazine 22(1) (2001) 73-84. 